\title{
Pending Problems in QSOs
}

\author{
Martín López-Corredoira ${ }^{1,2}$ \\ ${ }^{1}$ Instituto de Astrofisica de Canarias, La Laguna, Spain \\ ${ }^{2}$ Departamento de Astrofisica, Universidad de La Laguna, La Laguna, Spain \\ E-mail:martinlc@iac.es \\ Received February 3, 2011; revised March 17, 2011; accepted April 11, 2011
}

\begin{abstract}
Quasars (Quasi Stellar Objects, abbreviated as QSOs) are still nowadays, close to half a century after their discovery, objects which are not completely understood. In this brief review a description of the pending problems, inconsistencies and caveats in the QSO's research is presented. The standard paradigm model based on the existence of very massive black holes that are responsible for the QSO's huge luminosities, resulting from to their cosmological redshifts, leaves many facts without explanation. There are several observations which lack a clear explanation, for instance: the absence of bright QSOs at low redshifts, a mysterious evolution not properly understood; the inconsistencies of the absorption lines, such as the different structure of the clouds along the QSO's line of sight and their tangential directions; the spatial correlation between QSOs and galaxies; and many others.
\end{abstract}

Keywords: Quasars, General-Cosmology, Miscellaneous

\section{Introduction, QSOs: Observations, Standard View}

The topic of this article is the objects called Quasars (contraction of QUASi-stellAR radio source) or QSOs (Quasi-Stellar Objects). The first series of these objects were discovered with radio telescopes in the late 1950s. Many were recorded as radio sources with no corresponding visible object, hence their name "quasar", but optical counterparts were discovered later. Indeed, only around $10 \%$ of these objects have strong radio emission ('radioloud') [1]; hence it would be better to use the name "QSO" to refer to them, including the "radio-loud" and the 'radio-quiet' classes, although the name "quasar" is also used for radio-quiet QSOs.

Their radiation is emitted across the spectrum from $\mathrm{X}$-rays to the far-infrared with a peak in the ultravioletoptical bands, with some quasars also being strong sources of radio emission and of gamma-rays. Some quasars display rapid changes in luminosity in the optical and even more rapidly in the X-rays. Their spectra is characterized by a high redshift and a combination of very broad lines, of several percent of the speed of light, with narrow "forbidden" lines. Emission lines of hydrogen, mainly in the Lyman series and Balmer series, Helium, Carbon, Magnesium, Iron and Oxygen are the brightest lines. The atoms emitting these lines range from neutral ones to those having a degree of ionization higher than the possible ionizations produced by star radiation. In early optical images, quasars looked like point sources, indistinguishable from stars, except for their peculiar spectra. With infrared telescopes and the Hubble Space Telescope, the "host galaxies" surrounding the quasars have been identified in some cases.

According to the standard view, a QSO is an extremely powerful and distant active galactic nucleus. Their redshift is believed to be cosmological, associated to the expansion of the Universe, so their high redshift implies long distances, and consequently, huge luminosities. These objects are thought to be comprised of compact regions of $10-10,000$ Schwarzschild radii across surrounding the central supermassive black hole of a galaxy. The huge luminosity of quasars results from the accretion discs of central supermassive black holes, which can convert on the order of $10 \%$ of the mass of an object into energy as compared to $0.7 \%$ for the p-p chain nuclear fusion process that dominates the energy production in sun-like stars. The widths of the broad lines resulting from Doppler shifts are due to the high speeds of the gas emitting those spectral lines. Fast motions strongly would indicate a large mass. Since they cannot continue to feed at high rates for 10 billion years, after the accretion of the surrounding gas and dust is terminated, they would become ordinary galaxies, in few tens of Myr. Unified 
models were developed in which QSOs were classified as a particular kind of an active galaxy, like a Seyfert 1 but with higher luminosity due to the higher mass of the black hole and a general consensus emerged. In many cases it is simply based in the viewing angle that distinguishes them from other classes of active galaxies, such as Seyfert 2, blazars, radio galaxies.

There are plenty of books and reviews about these fascinating objects and the standard hypothesis to interpret them [2-4]. In this review I pretend to do something different: rather than presenting the successes of the standard theory in our understanding of the QSOs, I want to show the dark side, the aspects of which are still not very clear and deserve further consideration, either for improving the present standard theory, or to modify it, and even to change it completely if it were necessary. This article is not a forum for the discussion of all the possible theoretical approaches, but deals with the observational facts which could affect the discussion of what is known and/or what is still unknown.

Quasars (Quasi Stellar Objects, abbreviated as QSOs) are still nowadays, close to half a century after their discovery, objects which are not completely understood. In this brief review a description of the pending problems, inconsistencies and caveats in the QSO's research is presented. The standard paradigm model based on the existence of very massive black holes that are responsible for the QSO's huge luminosities, resulting from their cosmological redshifts, leaves many facts without explanation. Possibly not all of the cited references of this review are correct. My task here is just to compile the bibliography on the pending problems, not to critically examine them. This review is not complete, there are indeed hundreds or thousands of references relevant to these questions, although I think the references hereby presented are quite representative. Nonetheless, given the sort of the material displayed in our references, one can get a general glimpse of what are the most relevant topics discussed nowadays pertaining to the nature of QSOs.

\section{Very High Luminosity at High Redshift}

As said previously, the most remarkable characteristic of QSOs is perhaps their very high luminosity. The luminosities of the brightest QSOs, using the standard interpretation, are as bright as several thousands of $\mathrm{cD}$ galaxies (the brightest galaxy in a cluster of galaxies at low redshift $z$ ). Only one QSO may be as bright as tens of large clusters of galaxies (with $\sim 1000$ large galaxies in each of them) in a relatively compact region. The regions should be very compact in order to justify their strong variability in short times.

To reduce their luminosity, some prefer to think that there is a magnification due to gravitational lenses, but no evidence of that was found. Yamada et al. [5] or Richards et al. [6] examined the fields of some QSOs at $z$ $\approx 6$ and concluded that they are not gravitationally magnified, so the luminosity at that redshift must be very high. The anisotropy of the QSO radiation, observable only when the beam is pointed towards us, would also reduce the luminosity; but the number of sources would be much higher, a huge number, and we would then see a large number of them in the nearby Universe, even if their beams of maximum flux are not pointing towards us; local AGNs (Active Galactic Nuclei) would experience also this strong anisotropy radiation emission. It is not the case, we do not observe that. Therefore, the luminosity of the QSOs must be really huge, provided that their assumed distance is correct.

Since their discovery, long debates have taken place on whether the distance pointed by the redshift is real or not. After that period of debate in the $60 \mathrm{~s}$ and the early $70 \mathrm{~s}$, the mainstream of astronomers adopted the consensus that the redshift of the QSOs is cosmological in origin and therefore the luminosity is intrinsically very high. Hence, it could be explained in terms of supermassive black holes (e.g., [4], ch. 5; [7]) while discarding other alternative interpretations. Nonetheless, some apparent inconsistencies remain still within the standard explanation.

In the case of ultramassive black holes of around $10^{10}$ $\mathrm{M}_{\mathrm{Sun}}$, necessary to explain the extremely high luminosities of high redshift QSOs, they would attract the surrounding material at relativistic speeds and would become strongly redshifted [8], something which is not observed. Some physical variables should be proportional to the distance of a source, such as the Faraday rotation or the time dilation factor, but they are not observed to be correlated to the redshift. The polarization of radio emission rotates as it passes through magnetized extragalactic plasmas. Such Faraday rotations in QSOs should increase (on average) with distance. If redshift indicates distance, then rotation and redshift should increase together. However, the mean Faraday rotation is less near $z=2$ than near $z=$ 1 [9]. Time dilation, which is observed in supernovae, should also be observed in QSOs, increasing the periods of variability with the distance, but it is not observed in QSOs against expectations [10].

Moreover, the huge dispersion in the magnitude-redshift relation for QSOs [11] makes impossible to derive a Hubble law for them. This is not a strong argument since the intrinsic dispersion of luminosities might be high itself, but it might be pointing out that something is wrong with the distance measurement.

\section{Host Galaxies}

The luminosity of the host galaxies, which are supposed 
to be normal galaxies and whose luminosity come only from the stellar emission, have also extremely high luminosities. Schramm et al. [12] detected host galaxies at $z=3$ which are extremely luminous: down to $M_{V, r e s t}=$ -26.4 , that is, $L_{V, r e s t} \approx 3 \times 10^{12} L_{V, \text { Sun }}$. Its color $(B-V)_{\text {rest }} \approx$ 0.0 indicates a young population $(0.3 \mathrm{Gyr})$, so its stellar mass is $M_{*} \sim 5 \times 10^{11} M_{\text {Sun }}$ [12], somewhat high, although within the possible values. Therefore, the explanation for these high luminosities is that we could be observing a very young populations of stars.

Magain et al. [13] reported on the observation of a quasar lying at the edge of a gas cloud, whose size is comparable to that of a small galaxy, but whose spectrum shows no evidence for stars. The gas cloud is excited by the quasar itself. Magain et al. could not see any host galaxy in it; if a host galaxy were present, it should be at least six times fainter than it would normally be expected to be for such a bright quasar. This tells us that the host galaxies, although they are normally brighter than normal galaxies, in some cases are much fainter or inexistent. We do not know the reason.

Other problems to solve in the host galaxies remain: the dynamical mass of molecular gas of a case at $z=6.4$ $\left(\sim 5.5 \times 10^{10} M_{\text {Sun }}\right)$ is too high to leave room for other kinds of matter, and it cannot accommodate the predicted $10^{12} M_{\text {Sun }}$ stellar bulge necessary for its massive black hole [14]. There are also unexpected non-detections of cold neutral gas in the host galaxies of high redshift QSOs (at $>10^{23} \mathrm{~W} / \mathrm{Hz} ;[15]$ ).

\section{Age and Metallicity of High Redshift QSOs}

Some QSOs are apparently somewhat older than the Universe at their corresponding redshift. For instance, the quasar APM $08279+5255$ at redshift $z=3.91$ has an age of 2 - 3 Gyr, which constrains $\Omega_{m}$ to be less than 0.21 [16], lower than the accepted values for the standard cosmology nowadays. Possibly the age measurement is somewhat overestimated and this would explain the inconsistency, but it is important to bear in mind that there are pending cases like this to be solved.

Big Bang requires that stars, QSOs and galaxies in the early universe be "primitive", meaning mostly metal-free, because it requires many generations of supernovae to build up metal content in stars. But the observations show the existence of even higher than solar metallicities in the "earliest" QSOs and galaxies [16-20]. The iron to magnesium ratio increases at higher redshifts [21]. And what is even more amazing: there is no evolution of some line ratios, including iron abundance [22-25] between $z=0$ and $z=6.5$, iron abundance at $z \sim 6$ QSOs is similar to its abundance in local QSOs. The amount of dust in high redshift galaxies and QSOs is also much higher than expected [26]. In view of these evidences, orthodox cos- mologists claim now that the star formation began very early and produced metals up to the solar abundance quickly, in roughly half Gyr. However, it is not enough to come up with such a surprising claim, it needs to be demonstrated, and I do not see any evidence in favor of such a quick evolution in the local galaxies.

\section{Evolution or Non-Evolution of QSOs}

There is another remarkable fact about the luminosity of QSOs. They are extremely bright at high redshift, but QSOs at low redshift have got a much lower luminosity. From the analysis of the bolometric luminosity function of QSOs at different redshift [27], it is clear that the relative abundance of high luminosity QSOs decreases quickly at low redshift. In visible, below $z=0.3$, the rate of luminosity decrease begins to slow down and below $z=0.1$ the luminosity begins to increase again [28]. At SDSS survey, all QSOs at $z<0.4$ are fainter than $M_{B}=-26$ (with K-corrections) while there are plenty of QSOs tens of times brighter than this limit at higher redshifts. Also in other wavelengths this fact is observed clearly: in the $\mathrm{X}$-ray region it is particularly strong the evolution at low redshift [29]; or in the radio regime ([30,31], Figures 9 and 10). A strong density and luminosity evolution is required. It seems that we live in the era in which the bright QSOs have disappeared.

It is usual to claim that evolution is the wild card which solves this kind of problems. Something very different should have happened at high redshift with respect to the low redshift Universe to obtain this different level of luminosity. However, no visible signs of this evolution are observed. There is no indication of any significant evolution in the X-ray properties of quasars between redshifts 0 and 6 , apart from the intrinsic luminosity, suggesting that the physical processes of accretion onto massive black holes have not changed over the bulk of cosmic time [32]. Also, the spectral features of low and high redshift QSOs are very similar [33]. There are not variations of black hole masses and Eddington ratios for equal luminosity QSOs [34]. Therefore, the situation is that QSOs have a strong evolution in the values of their luminosity but not significant change in other properties, and it is not well understood which is the cause of the luminosity evolution. Possibly the environment might change. Nonetheless, do we know the connection between the triggering of activity and the environment?

\section{Triggering of Activity}

It is usually suggested that the interactions with the companion galaxies are related to the mechanism of feeding the black hole of the QSO [35,36]. Horst \& Duschl [37] pre- 
sented the results of an extremely simple cosmological model combined with an evolutionary scenario in which both the formation of the black hole as well as the gas accretion onto it are triggered by major mergers of gas-rich galaxies. Despite the very generous number of approximations their model reproduces the quasar density evolution in remarkable agreement with some observations. However, other authors [8] find difficulties to understand how the very massive black holes are formed. Kundt [8] thinks that centrifugal forces, pressures, and detonations prevent huge amounts of material to be collapsed.

Many of the QSO host galaxies at low redshift suffered mergers with accompanying starbursts [38,39]. Jahnke et al. [38] showed that $\approx 50 \%$ of the host galaxies show distortions in their rotation curves or peculiar gas velocities above normal maximum velocities for disks, sign of mergers. And all host galaxies have quite young stellar populations, typically $1-2 \mathrm{Gyr}$. While this presents evidence for a connection of galaxy interaction and AGN activity for half of the sample, this is not clear for the other half. There is $\mathrm{a} \approx 50 \%$ of the host galaxies which are undistorted disk dominated. Bennert et al. [40] think that most QSO host galaxies experienced mergers with accompanying starbursts but that the activity is triggered with a delay of several hundreds Myr after the merger. Even so, why don't we see evidence of mergers in many host galaxies?

The relationship of AGN triggered by mergers is not so clear, there are many observations apparently pointing to the opposite direction. In fact, Coldwell \& Lambas [41] have shown that quasars at $z<0.2$ systematically avoid high density regions, living in regions less dense than cluster environments. The environment of QSOs is populated by galaxies systematically bluer, and preferentially with disk-type morphology. And at $0.5 \leq z \leq 0.8$, only $10 \%$ of QSOs live in relatively rich clusters of Abell richness class $1-2$, and $45 \%$ of them live in field-like environments [42]. AGNs live also in low-density regions $[43,44]$, in even lower density regions than QSOs [45]; and QSOs with low mass black holes are in lower density regions than those with high mass black holes [45]. Unless the velocity in a cluster of galaxies is so high that strongly reduces the formation of mergers, the number of mergers should be higher in richer environments and it would lead to a lower triggering of activity and starbursts in low dense regions. We might also consider that galaxies in rich clusters are stripped of their interstellar medium by harrasment, so it would be reasonable that the QSO activity is less than in the field galaxies, but the ratio of spiral galaxies with non-stripped gas is still high enough to consider there should be activity triggering. More recently, Cisternas et al. [46] have shown directly that there is not an enhanced frequency of major merger signatures for the AGN hosts with respect to other galaxies, so this points out that major mergers should not be an important element for the triggering of activity.

There is evidence for a significant post-starburst population in many luminous AGNs, and that a direct, causal link might exist between star formation and black hole accretion [47]. The detection of large amounts of warm, extended, molecular gas also points that QSOs have vigorous star formation [14]. However, it is also common nowadays the proposal that AGN host galaxies are a transition population, being the AGNs the mechanism for star-formation quenching, where the black hole blows out the gas.

Therefore, to sum up this section, we have no idea of the mechanism which triggers the activity in galaxies, and the different observations point to different directions within the actual proposed scenarios.

\section{Superluminal Motions}

Superluminal motions of sources at high distance $(D)$ are observed, i.e. angular speeds $\omega$ between two radio emitting blobs which imply linear velocities $v=D \omega$ greater than the speed light [48]. For instance, the QSO 0805077 presents apparent superluminal motions up to 59.1 $h^{-1} c$ [49].

There are some explanations. The so called relativistic beaming model [50] assumes that there is one blob $A$ which is fixed while blob $B$ is traveling almost directly towards the observer with speed $V<c$ with an angle $\cos ^{-1}(V / c)$ between the line of motion and the line $B$-observer. This leads to an apparent velocity of separation which may be greater than $c$. There is also another proposal in a gravitational bending scenario [51]. However, both explanations share the common criticism of being contrived and having somewhat low probability $\left(\sim 10^{-4}\right)$ [52]. In the case of blazars, the superluminal motions in blazars can be statistically explained in the frame of the unification scheme of AGNs [53].

\section{Periodicity of Redshifts}

Another problem with QSOs which has a long history and without a clear agreement is the periodicity of redshifts. In a homogeneous and isotropic universe we expect the redshift distribution of extragalactic objects to approximate a continuous and aperiodic distribution. However, a periodicity with $\Delta z=0.031$ or 0.062 was found for the QSOs [54-56], which cannot be understood in terms of the Cosmological Hypothesis. Other authors found a periodicity of QSOs with a period of 0.089 the function $\log (1+z)$ instead of regular intervals linear in $z[57,58]$.

However, Hawkins et al. [59] and Tang \& Zhang 
$[60,61]$ found that there is no periodicity of QSOs in SDSS and 2dF surveys beyond randomness and selection effects. Napier \& Burbidge [62] argued that Hawkins et al. had not measured the redshifts of these faint quasars with respect to the redshift of their active parent galaxies. The periodicity found of the redshifts is measured with respect to the parent galaxy; people who do not find a periodicity would simply measure the redshift with respect to us $(z=0)$ - say Napier \& Burbidge. Therefore, the debate has not ended and it is not clear who is right.

\section{Correlation with Galaxies of Lower Redshift}

There are several statistical analyses [63-70] displaying an excess of high-redshift sources near the low-redshift galaxies, or positive and very significant cross correlations among surveys of galaxies and QSOs, or an excess of pairs of QSOs with very different redshifts, etc.

There are plenty of individual cases of galaxies with an excess of QSOs with high redshifts near the centre of nearby galaxies, mostly AGNs [66,71-75]. In some cases, QSOs are only a few arc-seconds away from the centre of the galaxies. Examples are NGC 613, NGC 1068, NGC 1097, NGC 3079, NGC 3842, NGC 6212, NGC 7541, NGC 7319 (separation galaxy/QSO: 8"), 2237 + 0305 (separation galaxy/QSO 0.3"), 3C 343.1 (separation galaxy/ QSO: 0.25"), NEQ3, etc. In some cases there are even filaments/bridges/arms apparently connecting objects with different redshift: in NGC 4319 + Mrk 205, Mrk273, QSO1327-206, NGC $3067+3 \mathrm{C} 232$ (in radio), NGC 622, NGC 3628 (in X-ray and radio), ESO 1327-2041 + QSO 1327-206, 4C17.09, UGC 892, NEQ3, etc. The probability of chance projections of background/foreground objects within a short distance of a galaxy or onto the filament is very low (down to $10^{-8}$ or even lower). The alignment of sources with different redshifts also suggests that they may have a common origin, and that the direction of alignment is the direction of ejection. This happens with some configurations of QSOs around 1130+106, 3C212, NGC 4258, NGC 2639, NGC 4235, NGC 5985, GC 0248+430, etc. Another observation suggesting the association QSO/ galaxies with different redshift is that no absorption lines were found in QSOs corresponding to foreground galaxies (e.g. PKS $0454+036$, PHL 1226), or distortions in the morphology of isolated galaxies.

The standard consensus is that all these cases are just random projections of background/foreground objects rather than the real associations of objects with different redshifts. This might be true in many cases, but the statistics still shows an excess number compared to the expected values for random projections. Hence, it remains difficult to explain these results in terms of random pro- jections. Typical rebuffs such as "it is an a posteriori statistical calculation" or other considerations such as a bias, incompleteness, gravitational lensing, do not solve the anomalies in general $[75,76]$. On the other hand, the main supporters of the hypothesis of non-cosmological redshifts continue to produce tens of analyses of cases in favor of their ideas without too much care, pictures without rigorous statistical calculations in many cases, or with wrong identifications, underestimated probabilities, biases, use of incomplete surveys for statistics, etc., in many other cases. Some cases which were claimed to be anomalous in the past have found an explanation in standard terms [76]. There are, however, many papers in which no objections are found in the arguments and they present quite controversial objects, but due to the bad reputation of the topic, the community simply ignores them. This has become a topic in which everybody has an opinion without having read the papers or knowing the details of the problem, because some leading cosmologists have said it is bogus. Therefore, despite the many efforts by most cosmologists to forget this old problem encountered with QSOs, the unexplained data are still there pointing out to us that we do not understand these phenomena completely. I maintain a neutral position, neither in favor of nor against non-cosmological redshifts.

\section{Emission Lines}

The standard model assumes that QSOs are the same type of objects as Seyfert 1 galaxies but much brighter. Both of them present the characteristic broad emission lines for hydrogen, carbon and other elements, together with a narrow emission of "forbidden" lines in the case of elements like oxygen, nitrogen, sulfur, etc. According to the standard black hole scenario and its accretion disc (e.g., [4], ch. 5), the broad lines stem from the inner region with a strong velocity dispersion, while the forbidden lines would be generated in the outer regions. These lines depend on the physical conditions of the clouds and the spectral energy distribution that photo-ionizes the clouds. Although this scenario explains the main basic spectral features, it remains to clarify some detailed observations. For instance, some analyses of the spectra are given by Sulentic [77], who believes that the doublepeaked Balmer emission lines are better fitted with the bi-cone outflow model $[78,79]$ rather than the model of accretion disks, or perhaps a combination of accretion disks and outflows. Indeed, the double-peaked optical emission lines are present only in $\sim 5 \%$ of the AGNs, which raises another problem in supporting the black holes hypothesis as the engines of the activity.

According to the unification model, the differences between narrow-line AGNs (Seyfert 2) and broad-line 
ones (Seyfert 1) stem from the orientation of the toroidal regions of large extinction in the same type of galaxies. But the relative ratios of narrow and broad line AGNs cannot be reconciled with this simple model of unification in which the tori have the same optical depth and their opening angle is found to be independent of the luminosity [80]; it requires a modification of the tori's extinction and/or cone angles for objects of different luminosity. Among the spiral AGNs with close satellite companions, only a $2.6 \%(1 / 39)$ of them are Seyfert 1 [81]; this cannot be explained either by a simple unification model and requires modifications in terms of extra extinctions in presence of companions. Other observations, pointing out that the differences between Seyfert 2 and Seyfert 1 cannot be due entirely to different orientations of the same object, can also be found in the literature (e.g., [4], sect. 12.6.5). Hence, it is required, in general, a revision of the simple unification model. This does not mean that the main aspects of the unification model are wrong, but there are many observations which do not fit its predictions unless the model is made more complex with the introduction of more ad hoc terms.

\section{Absorption Lines}

According to the standard interpretation, the absorption lines in QSOs are produced by the footprint in the intergalactic medium that the paths of the photons take. The redshift is associated to the path of the photons taken across the cloud. However, some observations are not always consistent with this scenario. For instance, the HST NICMOS spectrograph has searched for objects associated to the absorption lines of damped Ly- $\alpha$ systems (DLAs) of some QSOs directly in the infrared, but failed for the most part to detect them [82]. Moreover, the relative abundances of DLAs have a surprising uniformity, unexplained in the standard model [83], except for the clouds which have a velocity difference less than 6000 $\mathrm{km} / \mathrm{s}$ from a QSOs, where the excess density by a factor 2 (at 3.5\%) [84] is something which has neither a clear interpretation, unless the result by Russell et al. is a random fluctuation (it is only $3.5 \sigma$ ).

The Ly- $\alpha$ forest is supposed to be produced by hydrogen in clouds of the intergalactic medium along the line of sight. The temperature of these clouds does not change with redshift [85], a fact which does not fit the normal predictions of the standard model since the density of the clouds should have changed along the history of the Universe. There is not clustering of the clouds or it is very weak [86], contrary to what it would be expected.

Hennawi \& Prochaska [87] used a sample of 17 Lyman limit systems with column density $N_{H I}>10^{19} \mathrm{~cm}^{-2}$ selected from 149 projected quasar pair sightlines, to in- vestigate the clustering pattern of optically thick absorbers around luminous quasars at $z \approx 2.5$. Specifically, they measured the quasar-absorber correlation function in the transverse direction, and found a comoving correlation length of $r_{0}=9.2+1.5-1.7 \mathrm{Mpc} / \mathrm{h}$ (comoving) assuming a power law correlation function with exponent $\gamma=$ 1.6. Applying this transverse clustering strength to the line-of-sight would predict that $\sim 15 \%-50 \%$ of all quasars should show a $N_{H I}>10^{19} \mathrm{~cm}^{-2}$ absorber within a velocity window of $v<3000 \mathrm{Km} / \mathrm{s}$. This value overpredicts the number of absorbers along the line-of-sight by a large factor, providing compelling evidence that the clustering pattern of optically thick absorbers around quasars is highly anisotropic. Hennawi \& Prochaska [87] have argued that the most plausible explanation for the anisotropy is that the transverse direction is less likely to be illuminated by ionizing photons than the line-of-sight direction, and that absorbers along the line-of-sight are being photo-evaporated. An unbelievable explanation which serves to hide the fact that we have an unexpected observation within the standard interpretation of the origin of these absorption lines in QSOs.

Prochter et al. [88] report on a survey for strong intervening MgII systems along the sight-lines of long-duration gamma-ray bursts (GRBs). The roughly four times higher incidence along the GRB sight-lines than in QSOs sightlines with the same redshift is inconsistent with a statistical fluctuation by greater than $99.9 \%$ C.L. leading to a lower observed incidence along quasar sight-lines. Prochter's et al. [88] analysis of these results suggests that at least one of our fundamental beliefs on the absorption-line research is flawed. Tejos et al. [89] confirmed it but with a factor three in the incidence and only MgII systems having equivalent width at rest larger than 1 angstroms. However, Tejos et al. [90] and Sudilovsky et al. [91] conducted a similar study using CIV absorbers with GRB systems and their column density distribution and number density of this sample do not show any statistical differences with the same quantities measured in the QSO spectra. Maybe the discrepancy stems from a higher dust extinction in the strong MgII QSO samples studied up to now [91]. Frank et al. [92] propose that the solution is that the QSO beam size is 2 times larger than the GRB beam sizes on average. Porciani et al. [93] have argued that the combined action of some effects can substantially reduce the statistical significance of the discrepancy. Possibly this discrepancy can be solved in standard terms but it is not certain.

\section{Conclusions}

Irrespective of who is right or wrong, either the researchers who are following the standard interpretation of QSOs 
or the others who are following a different one, the general impression which emerges from all of these problems is that we do not yet understand very well many aspects of these objects and further research is still necessary.

If I had to express my particular opinion, I would say that the three most puzzling points are: 1) the total absence of bright QSOs at low redshift, a mysterious evolution not properly understood; 2) the inconsistencies of the absorption lines, such as the different structure of the clouds, when performing comparisons between measurements along the tangential and the line of sight of QSOs; 3) the spatial correlations among QSOs and nearby galaxies.

Nonetheless, one must not forget that there are also good reasons to support the standard scenario of QSOs, particularly the results about the large distances and luminosities. Just to select three among them: 1) the association of host galaxies with their QSOs shows that the luminosity of the central part of the object is much higher than the rest of the galaxy, and the hosts have angular sizes decreasing with redshift; 2) the absorption lines in many cases have a successful interpretation in terms of gas or galaxies intervening along the line of sight; 3) cases involving gravitational lensing indicate that the distance of QSOs is much higher than the distance of the lensing galaxy. And, within the large distance/luminosity assumption, there are also good reasons to support the standard paradigm model based on the existence of very massive black holes with their accretion discs.

It would be desirable that we could proclaim that we understand everything related to these fascinating objects before other 50 years went by. But up to now we should leave at least some room for more discussions and even having an open mind to embrace novel hypotheses in the interpretation of QSOs.

\section{Acknowledgements}

Thanks are given to Carlos Castro Perelman for proofreading this paper.

\section{References}

[1] W. H. de Vries, R. H. Becker and R. L. White, "Double-Lobed Radio Quasars from the Sloan Digital Sky Survey," The Astronomical Journal, Vol. 131, No. 2, 2006, pp. 666-679.

[2] M. J. Rees, "Black Hole Models for Active Galactic Nuclei," Annual Review of Astronomy and Astrophysics, Vol. 22, 1984, pp. 471-506.

[3] R. Antonucci, "Unified Models for Active Galactic Nuclei and Quasars," Annual Review of Astronomy and Astrophysics, Vol. 31, 1993, pp. 473-521.

[4] A. K. Kembhavi and J. V. Narlikar, "Quasars and Active Galactic Nuclei," Cambridge University Press, Cambridge,
1999.

[5] S. F. Yamada, Y. Shioya, Y. Taniguchi, et al., "Are Two z 6 Quasars Gravitationally Lensed?” Publications of the Astronomical Society of Japan, Vol. 55, No. 4, 2003, pp. 733-738

[6] G. T. Richards, M. A. Strauss, B. Pindor, et al., "A Snapshot Survey for Gravitational Lenses among $\mathrm{z}>=4.0$ Quasars. I. The $\mathrm{z}>5.7$ Sample," The Astronomical Journal, Vol. 127, No. 3, 2004, pp. 1305-1312.

[7] S. G. Djorgovski, M. Volonteri, V. Springel, V. Bromm and G. Meylan, "The Origins and the Early Evolution of Quasars and Supermassive Black Holes," In: H. Kleinert, R. T. Jantzen and R. Ruffini, Eds., Recent Developments in Theoretical and Experimental General Relativity, Gravitation and Relativistic Field Theories (11th Marcel Grossmann Meeting), World Scientific Publishing, Singapore, 2008, pp. 340-367.

[8] W. Kundt, "Critical Thoughts on Cosmology," In: Cosmology and Gravitation: XIII Brazilian School on Cosmology and Gravitation (AIP Conference Proceedings), AIP, Melville, New York, Vol. 1132, 2009, pp. 288- 302.

[9] H. C. Arp, "Intergalactic Magnetic Field and the Distance of Quasars from Faraday Rotation," Physics Letters A, Vol. 129, No. 3, 1988, pp. 135-139. doi:10.1016/0375-9601(88)90129-6

[10] M. R. S. Hawkins, "On Time Dilation in Quasar Light Curves," Monthly Notices of the Royal Astronomical Society, Vol. 405, No. 3, 2010, pp. 1940-1946.

[11] A. Hewitt and G. Burbidge, "A New Optical Catalog of Quasi-Stellar Objects," Astrophysical Journal Supplement Series, Vol. 63, 1987, pp. 1-246. doi:10.1086/191163

[12] M. Schramm, L. Wisotzki and K. Jahnke, "Host Galaxies of Bright High Redshift Quasars: Luminosities and Colours," Astronomy \& Astrophysics, Vol. 478, No. 2, 2008, pp. 311-319.

[13] P. Magain, G. Letawe, F. Courbin, P. Jablonka, K. Jahnke, L. Wisotzki and G. Meylan, "Discovery of a Bright Quasar without a Massive Host Galaxy," Nature, Vol. 473, 2005, pp. 381-384. doi:10.1038/nature04013

[14] F. Walter, D. A. Riechers, C. L. Carilli, F. Bertoldi, A. Weiss and P. Cox, "High-Resolution CO Imaging of High-Redshift QSO Host Galaxies," In: A. J. Baker, J. Glenn, A. I. Harris, J. G. Mangum and M. S. Yun, Eds., From Z-Machines to ALMA: (Sub) Millimeter Spectroscopy of Galaxies (ASP Conference Series), ASP, San Francisco, Vol. 375, 2007, p. 182.

[15] S. J. Curran, M. T. Whiting and J. K. Webb, "Where Is the Cold Neutral Gas in the Hosts of High Redshift AGN?" Memorie della Società Astronomica Italiana, Vol. 79, 2008, p. 1113.

[16] D. Jain and A. Dev, "Age of High Redshift Objects-A Litmus Test for the Dark Energy Models," Physical Letters B, Vol. 633, No. 4-5, 2006, pp. 436-440. doi:10.1016/j.physletb.2005.12.007

[17] X. Fan, V. K. Narayanan, R. H. Lupton, et al., "A Survey of $z>5.8$ Quasars in the Sloan Digital Sky Survey. I. 
Discovery of Three New Quasars and the Spatial Density of Luminous Quasars at z 6," The Astronomical Journal, Vol. 122, No. 6, 2001, pp. 2833-2849.

[18] R. H. Becker, X. Fan, R. L. White, et al., "Evidence for Reionization at $\mathrm{z} \sim 6$ : Detection of a Gunn-Peterson Trough in a $\mathrm{z}=6.28$ Quasar," The Astronomical Journal, Vol. 122, No. 6, 2001, pp. 2850-2857.

[19] A. Constantin, J. C. Shields, F. Hamann, C. B. Foltz and F. H. Chaffee, "Emission-Line Properties of z $>4$ Quasars," Astrophysical Journal, Vol. 565, No. 1, 2002, pp. 50-62. doi:10.1086/324395

[20] L. E. Simon, F. W. Hamann and M. Pettini, "Physical Properties of Absorbers in High Redshift Quasars," In: R. Guzmán, C. Packham, J. M. Rodríguez-Espinosa and S. Torres-Peimbert, Eds., First Light Science with the GTC, Instituto de Astronomâia, Universidad Nacional Autâonoma de Mâexico, Miami, Vol. 29, 2007, p. 177.

[21] F. Iwamuro, K. Motohara, T. Maihara, M. Kimura, Y. Yoshii and M. Doi, "Fe II/Mg II Emission-Line Ratios of QSOs within $0<\mathrm{z}<5.3$," Astrophysical Journal, Vol. 565, No. 1, 2002, pp. 63-77.doi:10.1086/324540

[22] M. Dietrich, F. Hamann, I. Appenzeller and M. Vestergaard, "Fe II/Mg II Emission-Line Ratio in High-Redshift Quasars," Astrophysical Journal, Vol. 596, No. 2, 2003, pp. 817-829. doi: $10.1086 / 378045$

[23] W. Freudling, M. R. Corbin and K. T. Korista, "Iron Emission in z 6 QSOs," Astrophysical Journal, Vol. 587, No. 2, 2003, pp. L67-L70. doi:10.1086/375338

[24] R. Maiolino, Y. Juarez, R. Mujica, N. Nagar and E. Oliva, "Early Star Formation Traced by the Highest Redshift Quasars," Astrophysical Journal, Vol. 596, No. 2, 2003, pp. L155-L158. doi:10.1086/379600

[25] A. J. Barth, P. Martini, C. H. Nelson and L. C. Ho, "Iron Emission in the $\mathrm{z}=6.4$ Quasar SDSS J114816.64+ 525150.3," Astrophysical Journal, Vol. 594, No. 2, 2003, pp. L95-L98. doi:10.1086/378735

[26] L. Dunne, S. Eales, R. Ivison, H. Morgan and M. Edmunds, "Type II Supernovae as a Significant Source of Interstellar Dust," Nature, Vol. 424, 2003, pp. 285-287. doi:10.1038/nature01792

[27] P. F. Hopkins, G. T. Richards and L. Hernquist, “An Observational Determination of the Bolometric Quasar Luminosity Function," Astrophysical Journal, Vol. 654, No. 2, 2007, pp. 731-753. doi:10.1086/509629

[28] M. B. Bell, "Further Evidence That the Redshifts of AGN Galaxies May Contain Intrinsic Components," Astrophysical Journal, Vol. 667, No. 2, 2007, pp. L129-L132. doi:10.1086/522337

[29] S. Shen, S. D. M. White, H. J. Mo, W. Voges, G. Kauffmann, C. Tremonti and S. F. Anderson, "The Soft X-Ray Properties of Quasars in the Sloan Digital Sky Survey," Monthly Notices of the Royal Astronomical Society, Vol. 369, No. 4, 2006, pp. 1639-1653. doi:10.1111/j.1365-2966.2006.10463.x

[30] A. H. Bridle and R. A. Perley, "Extragalactic Radio Jets," Annual Review of Astronomy and Astrophysics, Vol. 22, 1984, pp. 319-358.
[31] M. B. Bell, "Evidence that Quasars and Related Active Galaxies are Good Radio Standard Candles and that They are Likely to Be a Lot Closer than Their Redshifts Imply," 2006. arXiv.org, astro-ph/0602242.

[32] C. Vignali, W. N. Brandt, D. P. Schneider and S. Kaspi, "X-Ray Lighthouses of the High-Redshift Universe. II. Further Snapshot Observations of the Most Luminous z > 4 Quasars with Chandra," Astrophysical Journal, Vol. 129, No. 6, 2005, pp. 2519-2530.

[33] L. E. Segal and J. F. Nicoll, "Cosmological Implications of a Large Complete Quasar Sample," Proceedings of the National Academy of Sciences of the United States of America, Vol. 95, No. 9, 1998, pp. 4804-4807. doi:10.1073/pnas.95.9.4804

[34] M. López-Corredoira and C. M. Gutiérrez, "On the nonevolution of black hole masses and Eddington ratios for QSOs," Monthly Notices of the Royal Astronomical Society, Submitted, 2011.

[35] A. Stockton, "Compact Companions to QSOs," Astrophysical Journal, Vol. 257, No. 1, 1982, pp. 33-39. doi:10.1086/159959

[36] G. Canalizo and A. Stockton, "Quasi-Stellar Objects, Ultraluminous Infrared Galaxies, and Mergers," Astrophysical Journal, Vol. 555, No. 2, 2001, pp. 719-743. doi: $10.1086 / 321520$

[37] H. Horst and W. J. Duschl, "A Simple Model for Quasar Density Evolution," In: Relativistic Astrophysics Legacy and Cosmology, Springer-Verlag, Berlin, 2008, p. 224.

[38] K. Jahnke, L. Wisotzki, F. Courbin and G. Letawe, "Spatial Decomposition of on-Nucleus Spectra of Quasar Host Galaxies," Monthly Notices of the Royal Astronomical Society, Vol. 378, No. 1, 2007, pp. 23-40. doi:10.1111/j.1365-2966.2007.11623.x

[39] N. Bennert, G. Canalizo, B. Jungwiert, A. Stockton, F. Schweizer, C. Y. Peng and M. Lacy, "Evidence for Merger Remnants in Early-Type Host Galaxies of LowRedshift QSOs," Astrophysical Journal, Vol. 677, No. 2, 2008, pp. 846-857. doi:10.1086/529068

[40] N. Bennert, G. Canalizo, B. Jungwiert, A. Stockton, F. Schweizer, C. Y. Peng and M. Lacy, "Fueling QSOs: the Relevance of Mergers," Memorie della Società Astronomica Italiana, Vol. 79, 2008, pp. 1247-1250.

[41] G. V. Coldwell and D. G. Lambas, "Properties of Galaxies in Sloan Digital Sky Survey Quasar Environments at z $<0.2$," Monthly Notices of the Royal Astronomical Society, Vol. 371, No. 2, 2006, pp. 786-792. doi:10.1111/j.1365-2966.2006.10712.x

[42] M. Wold, M. Lacy, P. B. Lilje and S. Serjeant, "QSO Environments at Intermediate Redshifts,” In: I. Márquez, J. Masegosa, A. del Olmo, L. Lara, E. García and J. Molina, Eds., QSO Host and Their Environments, Kluwer Academic/Plenum Publishers, New York, 2001, p. 33.

[43] P. B. Westoby, C. G. Mundell and I. K. Baldry, "Are Galaxies with Active Galactic Nuclei a Transition Population?" Monthly Notices of the Royal Astronomical Society, Vol. 382, No. 4, 2007, pp. 1541-1551. doi:10.1111/j.1365-2966.2007.12553.x 
[44] A. Constantin, F. Hoyle and M. S. Vogeley, "Active Galactic Nuclei in Void Regions," Astrophysical Journal, Vol. 673, No. 2, 2008, pp. 715-729. doi:10.1086/524310

[45] N. E. Strand, R. J. Brunner and A. D. Myers, "AGN Environments in the Sloan Digital Sky Survey. I. Dependence on Type, Redshift, and Luminosity," Astrophysical Journal, Vol. 688, No. 1, 2008, pp. 180-189. doi:10.1086/592099

[46] M. Cisternas, K. J. Inskip, K. Jahnke, et al., "The Bulk of the Black Hole Growth Since $\mathrm{z} \sim 1$ Occurs in a Secular Universe: No Major Merger-AGN Connection," Astrophysical Journal, Vol. 726, No. 1, 2011, pp. 57-70. doi:10.1088/0004-637X/726/2/57

[47] L. C. Ho, "AGNs and Starbursts: What Is the Real Connection?" 2005, arXiv.org, astro-ph/0511157.

[48] M. H. Cohen, "Relativistic Motion in Quasars," In: S. L. Shapiro and S. A. Teukolsky, Eds., Highlights of Modern Astrophysics, Wiley Interscience, New York, 1986, p. 299.

[49] M. L. Lister, M. H. Cohen, D. C. Homan, et al., "MOJAVE: Monitoring of Jets in Active Galactic Nuclei with VLBA Experiments. VI. Kinematics Analysis of a Complete Sample of Blazar Jets," The Astronomical Journal, Vol. 138, No. 6, 2009, pp. 1874-1892.

[50] M. J. Rees, "Studies in Radio Source Structure-I. A Relativistically Expanding Model for Variable Quasi-Stellar Radio Sources," Monthly Notices of the Royal Astronomical Society, Vol. 135, 1967, pp. 345-360.

[51] S. M. Chitre and J. V. Narlikar, "On the Apparent Superluminal Separation of Radio Source Components," Monthly Notices of the Royal Astronomical Society, Vol. 187, 1979, pp. 655-659.doi:10.1007/BF02714473

[52] J. V. Narlikar and S. M. Chitre, "Faster-Than-Light Motion in Quasars," Journal of Astrophysics and Astronomy, Vol. 5, 1984, pp. 495-506.

[53] Y. Liu and S. N. Zhang, "The Lorentz Factor Distribution and Luminosity Function of Relativistic Jets in AGNs," Astrophysical Journal, Vol. 667, No. 2, 2007, pp. 724730. doi: $10.1086 / 520040$

[54] G. R. Burbidge and S. L. O'Dell, "The Distribution of Redshifts of Quasi-Stellar Objects and Related EmissionLine Objects," Astrophysical Journal, Vol. 178, 1972, pp. 583-606. doi:10.1086/151820

[55] M. B. Bell and D. McDiarmid, "Six Peaks Visible in the Redshift Distribution of 46,400 SDSS Quasars Agree with the Preferred Redshifts Predicted by the Decreasing Intrinsic Redshift Model," Astrophysical Journal, Vol. 648, No. 1, 2006, pp. 140-147. doi:10.1086/503792

[56] J. G. Hartnett, "Unknown Selection Effect Simulates Redshift Periodicity in Quasar Number Counts from Sloan Digital Sky Survey," Astrophysics and Space Science, Vol. 324, No. 1, 2009, pp. 13-16.

[57] K. G. Karlsson, "On the Existence of Significant Peaks in the Quasar Redshift Distribution," Astronomy \& Astrophysics, Vol. 58, No. 1-2, 1977, pp. 237-240.

[58] W. M. Napier, "Statistics of Redshift Periodicities," In: J.-C. Pecker and J. V. Narlikar, Eds., Current Issues in
Cosmology, Cambridge University Press, Cambridge, 2006, pp. 207-216.

[59] E. Hawkins, S. Maddox and M. Merrifield, "No Periodicities in 2dF Redshift Survey Data," Monthly Notices of the Royal Astronomical Society, Vol. 336, No. 1, 2002, pp. L13-L16. doi:10.1046/j.1365-8711.2002.05940.x

[60] S. M. Tang and S. N. Zhang, "Critical Examinations of QSO Redshift Periodicities and Associations with Galaxies in Sloan Digital Sky Survey Data," Astrophysical Journal, Vol. 633, No. 1, 2005, pp. 41-51. doi:10.1086/432754

[61] S. M. Tang and S. N. Zhang, "Evidence against NonCosmological Redshifts of QSOs in SDSS Data," In: D. Basu, Ed., Redshifts in Spectral Lines of Quasi Stellar Objects, Research Signpost, Kerala, 2010, pp. 125-136.

[62] W. Napier and G. R. Burbidge, "The Detection of Periodicity in QSO Data Sets," Monthly Notices of the Royal Astronomical Society, Vol. 342, No. 2, 2003, pp. 601-604. doi:10.1046/j.1365-8711.2003.06567.x

[63] Y. Chu, X. Zhu, G. Burbidge and A. Hewitt, "Statistical Evidence for Possible Association between QSOs and Bright Galaxies," Astronomy \& Astrophysics, Vol. 138, No. 2, 1984, pp. 408-414.

[64] X. F. Zhu and Y. Q. Chu, "The Association between Quasars and the Galaxies of the Virgo Cluster," Astronomy \& Astrophysics, Vol. 297, 1995, pp. 300-304.

[65] G. R. Burbidge, J. V. Narlikar and A. Hewitt, "The Statistical Significance of Close Pairs of QSOs," Nature, Vol. 317, 1985, pp. 413-415. doi:10.1038/317413a0

[66] G. R. Burbidge, "Noncosmological Redshifts," Publications of the Astronomical Society of the Pacific, Vol. 113, 2001, pp. 899-902. doi:10.1086/322152

[67] N. Benítez, J. L. Sanz and E. Martínez-González, "Quasar-Galaxy Associations Revisited," Monthly Notices of the Royal Astronomical Society, Vol. 320, No. 2, 2001, pp. 241-248. doi:10.1046/j.1365-8711.2001.03936.x

[68] E. Gaztañaga, "Correlation between Galaxies and QuasiStellar Objects in the Sloan Digital Sky Survey: A Signal from Gravitational Lensing Magnification?" Astrophysical Journal, Vol. 589, No. 1, 2003, pp. 82-99. doi: $10.1086 / 374616$

[69] J. G. Nollenberg and L. R. Williams, "Galaxy-Quasar Correlations between APM Galaxies and Hamburg-ESO QSOs," Astrophysical Journal, Vol. 634, No. 2, 2005, pp. 793-805. doi:10.1086/497103

[70] Y. L. Bukhmastova, "Quasars Lensed by Globular Clusters of Spiral and Elliptical Galaxies," Astronomy Letters, Vol. 33, No. 6, 2007, pp. 355-367. Translated from: Pi'sma v Astronomicheckii Zhurnal, Vol. 33, No. 6, 2007, pp. 403-416.

[71] H. C. Arp, "QSOs, Redshifts and Controversies," Interstellar Media, Berkeley, 1987.

[72] H. C. Arp, Catalogue of Discordant Redshift Associations, Apeiron, Montreal, 2003.

[73] M. B. Bell, "Further Evidence for Large Intrinsic Redshifts," Astrophysical Journal, Vol. 566, No. 2, 2002, pp. 705-711. doi:10.1086/338272 
[74] M. B. Bell, "On Quasar Distances and Lifetimes in a Local Model," Astrophysical Journal, Vol. 567, No. 2, 2002, pp. 801-810.doi:10.1086/338754

[75] M. López-Corredoira and C. M. Gutiérrez, "Research on Candidates for Non-Cosmological Redshifts," In: E. J. Lerner and J. B. Almeida, Eds., First Crisis in Cosmology Conference (AIP Conference Proceedings 822), AIP, Melville, New York, 2006, pp. 75-92.

[76] M. López-Corredoira, “Apparent Discordant Redshift Qso-Galaxy Associations," In: H. A. Harutyunian, A. M. Mickaelian and Y. Terzian, Eds., Evolution of Cosmic Objects through Their Physical Activity, (Viktor Ambartsumian's 100th anniversary), Gitutyun Publishing House of NAS RA, Yerevan, 2010, pp. 196-205.

[77] J. W. Sulentic, “Accretion Disks in Quasars?” In: J.-C. Pecker and J. V. Narlikar, Eds., Current Issues in Cosmology, Cambridge University Press, Cambridge, pp. 37-45.

[78] I. Goldman and J. N. Bahcall, "Asymmetric EmissionLine Regions with Out-Flowing Mass in QSOs and the $\mathrm{z} / \mathrm{ab} /$ at Least Equal to z/em/ Systems," Astronomy \& Astrophysics, Vol. 115, No. 2, 1982, pp. 242-248.

[79] A. Robinson, "On the Diversity of the Broad EmissionLine Profiles in Active Galactic Nuclei," Monthly Notices of the Royal Astronomical Society, Vol. 272, No. 3, 1995, pp. 647-664.

[80] A. Lawrence, "Classification of Active Galaxies and the Prospect of a Unified Phenomenology," Publications of the Astronomical Society of the Pacific, Vol. 99, 1987, pp. 309-334. doi:10.1086/131989

[81] D. Dultzin, J. J. González, Y. Krongold, et al., "Activity Induced by Gravitational Interaction in Galaxy Pairs. Mixed (E+S) Morphology Pairs," Memorie della Società Astronomica Italiana, Vol. 79, pp. 1326-1329.

[82] J. W. Colbert and M. A. Malkan, "NICMOS Snapshot Survey of Damped Lya Quasars," Astrophysical Journal, Vol. 566, No. 1, 2002, pp. 51-67. doi:10.1086/324779

[83] J. X. Prochaska and A. M. Wolfe, "The UCSD HIRES/ Keck I Damped Ly $\alpha$ Abundance Database. II. The Implications," Astrophysical Journal, Vol. 566, No. 1, 2002, pp. 68-92. doi: $10.1086 / 338080$

[84] D. M. Russell, S. L. Ellison and C. R. Benn, “An Excess of Damped Lyman $\alpha$ Galaxies Near Quasi-Stellar Objects," Monthly Notices of the Royal Astronomical Society,
Vol. 347, No. 1, 2006, pp. 412-422. doi:10.1111/j.1365-2966.2005.09964.x

[85] M. Zaldarriaga, L. Hui and M. Tegmark, "Constraints from the Ly $\alpha$ Forest Power Spectrum," Astrophysical Journal, Vol. 557, No. 2, 2001, pp. 519-526. doi: $10.1086 / 321652$

[86] A. Dobrzycki, J. Bechtold, J. Scott and M. Morita, "A Uniform Analysis of the Ly $\alpha$ Forest at $\mathrm{z}=0-5$. IV. The Clustering and Evolution of Clouds at $\mathrm{z}<=1.7$," Astrophysical Journal, Vol. 571, No. 2, 2002, pp. 654-664. doi: $10.1086 / 339983$

[87] J. F. Hennawi and J. X. Prochaska, "Quasars Probing Quasars. II. The Anisotropic Clustering of Optically Thick Absorbers around Quasars," Astrophysical Journal, Vol. 655, No. 2, 2007, pp. 735-748. doi:10.1086/509770

[88] G. E. Prochter, J. X. Prochaska, H.-W. Chen, et al., "On the Incidence of Strong Mg II Absorbers along GammaRay Burst Sight Lines," Astrophysical Journal, Vol. 648, No. 2, 2006, pp. L93-L96. doi:10.1086/508061

[89] N. Tejos, S. López, J. X. Prochaska, J. S. Bloom, H.-W. Chen, M. Dessauges-Zavadsky and M. J. Maureira, "Casting Light on the "Anomalous" Statistics of Mg II Absorbers Toward Gamma-Ray Burst Afterglows: The Incidence of Weak Systems," Astrophysical Journal, Vol. 706, No. 2, 2009, pp. 1309-1315. doi:10.1088/0004-637X/706/2/1309

[90] N. Tejos, S. López, J. X. Prochaska, H.-W. Chen and M. Dessauges-Zavadsky, "On the Incidence of C IV Absorbers Along the Sight Lines to Gamma-Ray Bursts," Astrophysical Journal, Vol. 671, No. 1, 2007, pp. 622627. doi: $10.1086 / 523088$

[91] V. Sudilovsky, S. Savaglio, P. Vreeswijk, C. Ledoux, A. Smette and J. Greiner, "Intervening Metal Systems in GRB and QSO Sight Lines: The Mg II and C IV Question," Astrophysical Journal, Vol. 669, No. 2, 2007, pp. 741-748. doi:10.1086/521525

[92] S. Frank, M. C. Bentz, K. Z. Stanek, M. Dietrich, C. S. Kochanek, S. Mathur and B. M. Peterson, "Disparate MG II Absorption Statistics towards Quasars and Gamma-Ray Bursts: A Possible Explanation," Astrophysics and Space Science, Vol 312, No. 3-4, 2007, pp. 325-330.

[93] C. Porciani, M. Viel and S. J. Lilly, "Strong Mg II Systems in Quasar and Gamma-Ray Burst Spectra," Astrophysical Journal, Vol. 669, No. 1, 2007, pp. 218-224. doi: $10.1086 / 512358$ 